\title{
Neue Nüchternheit und kritische Öffentlichkeit - die Niederlande und die europäische Integration
}

\author{
Alfred Pijpers*
}

Während des deutschen Ratsvorsitzes der Europäischen Union im ersten Halbjahr 2007 gedachte die Europäische Union im März mit einem Festakt der Unterzeichnung der Römischen Verträge vor fünfzig Jahren. ${ }^{1}$ Allerdings wurden die Feierlichkeiten von der Verfassungskrise überschattet, in die die Europäische Union durch das französische und niederländische „Nein“ zur europäischen Verfassung aus dem Frühjahr 2005 geraten war. Vor allem die Tatsache, dass zwei der Mitbegründer der Europäischen Verträge sich jetzt so abweisend zeigten, führte überall zu Erstaunen und Besorgnis. Wie ist die Situation in den Mitgliedstaaten, die so lange als treue Partner im europäischen Integrationsprozess gegolten haben, die doch beide einen ausgesprochen pro-europäischen Ruf hatten? Im Folgenden soll ein Versuch unternommen werden, diese Frage für die Niederlande zu beantworten.

Zu Beginn soll kurz auf die traditionelle, sehr gemeinschaftsfreundliche niederländische Haltung in der Europäischen Gemeinschaft und der Europäischen Union eingegangen werden. Daran schließt sich die Betrachtung darüber an, dass diese Haltung vor allem seit dem Beginn der 1990er Jahre nach und nach immer kritischer und pragmatischer wurde, bis hin zum Tiefpunkt der niederländischen Ablehnung des Europäischen Verfassungsvertrages am 1. Juni 2005. Dieses „Nein“ ist also nicht völlig unerwartet vom Himmel gefallen, obwohl es doch einen historischen Punkt in der niederländischen Europapolitik markiert. Im Anschluss daran wird argumentiert, dass die niederländische Ablehnung des Europäischen Verfassungsvertrages sowohl mit Veränderungen in der niederländischen wie in der europäischen Politik zusammenhängt. Eine Betrachtung der Position der heutigen Regierung Balkenende zum Mandat für eine neue Regierungskonferenz, die sich auch der Frage widmet, ob in den Niederlanden ein weiteres Referendum für die Ratifizierung eines Reformvertrages nötig ist, schließt diesen Beitrag ab.

\section{Die traditionelle niederländische Politik}

Die niederländische Ablehnung des europäischen Verfassungsvertrages und die offene Betonung der nationalen Eigenständigkeit und Interessen durch die derzeitige Regierung (siehe unten) bedeuten ohne Zweifel eine neue Phase in der niederländischen Europapolitik, vor allem im Vergleich zu den ersten Jahrzehnten des Integrationsprozesses. Als einem der Mitbegründer der Europäischen Verträge gehörten die Niederlande immer zu den Vorkämpfern zumindest der europäischen Wirtschaftsintegration. Die Niederlande waren über lange Jahre hinweg ein aktiver Fürsprecher eines gut funktionierenden gemeinsamen Marktes ohne Innengrenzen, auf der Basis einer gemeinschaftlichen Rechtsordnung mit zum Teil supranationalen Einrichtungen, mit einer gemeinsamen Währung und einer unabhängigen Zentralbank. Den Haag hat sich immer für den Beitritt neuer Mitgliedstaaten stark gemacht

* Dr. Alfred Pijpers, Netherlands Institute of International Relations „Clingendael“.

1 Der Text basiert auf einem Vortrag, den der Verfasser auf der wissenschaftlichen Konferenz ,50 Jahre Römische Verträge" gehalten hat. Die Konferenz wurde vom Institut für Europäische Politik in Zusammenarbeit mit dem Arbeitskreis Europäische Integration und dem Centre International de Formation Européenne in Berlin durchgeführt. 
und spielte als Ratsvorsitzender eine sehr konstruktive Rolle beim Zustandekommen wichtiger EU-Vertragsreformen, wie zum Beispiel die Einheitliche Europäische Akte und die Verträge von Maastricht und Amsterdam. Zusammen mit Belgien und Luxemburg bildeten die Niederlande oft eine Vorhut des Integrationsprozesses, die für den Rest der Europäischen Gemeinschaft und später der Europäischen Union Bahnbrechendes leistete im Bereich der Zollunion und des freien Personenverkehrs. Über die Jahrzehnte hinweg erfreuten sich die Niederlande eines ausgesprochen pro-europäischen Rufs.

Auch in der Innenpolitik war ,Europa“ über lange Zeit hinweg kaum ein Thema. Alle europäischen Grundverträge wurden über die Jahre hinweg von der Tweede und Eerste Kamer des Parlamentes mit großer Mehrheit angenommen und wurden integraler Bestandteil der nationalen Rechtsordnung. Nahezu alle politischen Parteien (mit Ausnahme der kleineren linken und rechten Parteien), die Sozialpartner und eine Vielzahl gesellschaftlicher Gruppierungen haben den europäischen Integrationsprozess üblicherweise in großen Linien unterstützt. In der Praxis war die europäische Zusammenarbeit in erster Linie eine Angelegenheit der Politik und der Wirtschaft sowie der amtlichen, diplomatischen und politischen Eliten in Den Haag und Brüssel, doch für den Prozess gab es in der Bevölkerung eine breite, wenn auch passive Unterstützung. Der Wetenschappelijke Raad voor het Regeringsbeleid (WRR, Wissenschaftlicher Beirat der Regierung) nannte dies ,einen wohlwollenden Konsens der breiten Masse“, vor allem, solange die europäische Integration dem Wohlstand zugutekam. ${ }^{2}$ Nur unzufriedene Bauern rührten sich von Zeit zu Zeit und im Hintergrund auch die Lobbys der Wirtschaft. Die Arbeitsgruppe Europa der Nationale Conventie (Nationalkonvent) schrieb 2006 in ihrem Teilbericht: „An der breiten demokratischen Unterstützung für die europäische Integration in den Niederlanden gab es in rund fünfzig Jahren kaum Zweifel. Auch nicht in Fragen des Euro oder der Erweiterung durch neue Mitgliedstaaten. Wer daran zweifelt, zweifelt auch an einem halben Jahrhundert niederländischer parlamentarischer Demokratie."3

Diese Aussage impliziert übrigens auch, dass die europäische Integration in den ersten Jahrzehnten vom größten Teil der Bevölkerung nicht als besonders bedrohlich für die nationale Identität erfahren worden sein kann. Im Gegenteil: „Der niederländische Wohlfahrtsstaat ist durch das Wachstum des europäischen Binnenmarktes und die Gelder, die dieser indirekt in die Staatskasse eingebracht hat, mit möglich gemacht worden. Dadurch konnte ein solides System der sozialen Sicherheit aufgebaut werden und gab es auch Geld für Bildung, Wohnungswesen, Gesundheitswesen etc. Auf diese Weise konnte Den Haag die Nation an sich binden und bewahrte so den vom Weltkrieg und der Dekolonisierung geschwächten niederländischen Staat vor dem Niedergang. “4 Europa wurde also jahrelang als eine wichtige Voraussetzung für die nationale politische Existenz und nicht als eine Bedrohung dafür gesehen.

2 Europa in Nederland. Rapport van de Wetenschappelijke Raad voor het Regeringsbeleid (WRR), Den Haag 2007, S. 39. Siehe auch Paul Dekker u.a.: Marktplaats Europa: vijftig jaar publieke opinie en marktintegratie in de Europese Unie, Den Haag 2007 (Europese Verkenning 5).

3 De Europese Unie als Statenverbond. Gedachten over de finaliteit van de Europese integratie, Den Haag 2006, S. 9. Die sogenannte Nationale Conventie (Nationalkonvent) wurde im Januar 2006 von der niederländischen Regierung ins Leben gerufen um zu untersuchen, wie das Vertrauen der Bürger in die niederländische Politik wieder hergestellt werden könnte. Es war ein breit zusammengesetztes Beratungsorgan mit einer separaten Arbeitsgruppe für die Europäische Union.

4 De Europese Unie als Statenverbond, 2006, S. 9. 


\section{Umbrüche}

Dieses Bild hat sich inzwischen stark verändert. Das „Nein“ bedeutet ganz bestimmt einen Umschwung des stillschweigenden Konsenses und die neuen, national gefärbten Politikakzente der Regierungen scheinen sich nur schwer zu verstehen mit der früher so offenen gemeinschaftlichen Mentalität. Dennoch dürfen die Trennlinien in dieser Hinsicht nicht zu scharf gezogen werden. Seit 2005 ist weniger von einem Trendbruch in der Politik als vielmehr von einem Stilbruch in deren Legitimierung die Rede. Die ruhmreiche gemeinschaftliche Orthodoxie wurde bereits in den achtziger Jahren mit allerlei pragmatischen und intergouvernementalen Elementen verwässert. Der Europäische Rat wird in Den Haag problemlos als Direktorium der Europäischen Union akzeptiert, auf Kosten der Position der Europäischen Kommission. Intensive bilaterale Kontakte und soft law haben die Verfahren der Gemeinschaft ergänzt und zum Teil sogar ersetzt. Das Verbessern der Position als Nettozahler wurde in den letzten Jahren sehr nachdrücklich als ein nationales Anliegen präsentiert. Während der zweiten Regierung Kok (1998-2002) stand in den Haager EU-Berichten: „Die Regierung ist sich bewusst, dass der nationale Staat noch immer wie kein anderer den Rahmen der Demokratie stellt. Allein schon aus diesem Grund richtet sich das Streben nicht auf die Erschaffung eines europäischen Superstaates. Die Mitgliedsstaaten bleiben das Fundament der Europäischen Union, auch in Zukunft. " 5

Sich Querzustellen ist keine Neuheit für ein Land, das bereits zu Beginn der 1960er Jahre die Pläne de Gaulles zur Politischen Union torpedierte und das bezüglich der Drogenpolitik ganz anders vorgeht, als die Mehrheit der Mitgliedstaaten. Die Niederlande haben sich seit jeher einer Mehrheitsbeschlussfassung im dritten Pfeiler widersetzt. Bereits früher scheiterte übrigens ein europäischer Vertrag durch niederländisches Zutun, nämlich als die Eerste Kamer am 19. Mai 1987 dem von den EG-Mitgliedstaaten beschlossenen Abkommen zur Errichtung einer Europäischen Stiftung die Zustimmung verweigerte. ${ }^{6}$ Dieses Abkommen sollte den Grundstein legen für ein Europäisches Kulturzentrum in Paris und hätte den Gnadenstoß für die in Amsterdam angesiedelte Europäische Kulturstiftung bedeutet.

Auch die Pläne für eine europäische Verfassung erhielten anfangs wenig Beifall. Joschka Fischers diesbezügliche Vorstellungen vom Mai 2000 wurden von Außenminister Jozias van Aartsen zunächst als eine Art Traumtänzerei abgetan. Er und andere Regierungsmitglieder brauchten keine föderalen Blaupausen und äußerten öffentliche Bedenken über die konstitutionellen Ambitionen der Europäischen Union. ${ }^{7}$ Auch unter Ministerpräsident Balkenende (seit 2002) ist dies der Fall. Minister Bernard Bot und Staatssekretär Atzo Nicolaï waren damals sicherlich keine begeisterten Anhänger des Verfassungsprojektes. Die Befürworter der europäischen Verfassung hatten sicherlich Recht mit dem Argument, dass während der Referendumskampagne eigentlich wenig Unterstützung von Regierungsseite kam.

Auch die öffentliche Meinung über die europäische Integration wurde stets skeptischer. Aus den Eurobarometer-Meinungsumfragen ergibt sich ab Mitte der 1990er Jahre ein deutlicher Rückgang der Unterstützung der europäischen Integration durch die niederländische Bevölkerung. ${ }^{8}$ Der Politikwissenschaftler Thomassen ist daher der Meinung, ,,dass es nicht stimmt, dass die Stimmung in den Niederlanden während des Referendums plötzlich umgeschlagen ist. Ein

5 De Staat van de Europese Unie 2001, Den Haag 2001, S. 17.

6 Handelingen Eerste Kamer 1986-1987, S. 30-1309, 1310, 19. Mai 1987, Stemming over het wetsvoorstel Goedkeuring van de op 29 maart te Brussel tot stand gekomen Overeenkomst tot oprichting van een Europese Stichting, met Regeling betreffende de instelling van een Voorbereidend Comité (17852).

7 Alfred Pijpers: Europese politiek in Nederlands vaarwater, Assen 2005, S. 154.

8 Für eine neuere Analyse siehe: Europa in Nederland, Den Haag 2007, S. 48-49. 
negativer Trend war bereits seit längerem zu beobachten. “9 Erwähnenswert ist auch die Untersuchung der Amsterdamer Soziologen Kapteyn und Schijf von 1996. Sie kamen zu dem Schluss, dass die niederländische Bevölkerung mehrheitlich für ,das Bewahren von Selbständigkeit und für weniger Integration“"war, ein Befund, der das Ergebnis des Referendums vorwegnimmt. ${ }^{10}$

\section{Das Warum des „Nein“ zum Verfassungsvertrag}

Es gibt viele Erklärungen für dieses „Nein“ (es richtete sich gegen die schnelle Erweiterung der Europäischen Union, gegen einen möglichen Beitritt der Türkei, gegen den teuren Euro, gegen die Politik der Regierung Balkenende, gegen die Globalisierung etc.) und zweifellos werden die Nein-Sager unterschiedliche Motive für ihre Ablehnung gehabt haben, aber im Grunde kann diese Ablehnung doch kaum anders gesehen werden denn als nationaler Widerstand gegen die wachsende Machtfülle Brüssels. Abgelehnt wurde ein Dokument, das ganz bewusst als eine „Verfassung für Europa“ vorgestellt wurde und das durch Symbolik und Inhalt auch ausdrücklich versuchte, den Grundstein für das zu legen, was der Vorsitzende des Europäischen Konvents, Valéry Giscard d'Estaing, selbst explizit als „Vereinigte Staaten von Europa“ bezeichnet hatte. In den Niederlanden wird dieser Begriff gegenwärtig nur noch von D66 verwendet, einer sozial-liberalen politischen Partei, die im Laufe von zehn Jahren von 24 auf drei Parlamentssitze in der Tweede Kamer geschrumpft ist. ${ }^{11}$

In einer neueren Untersuchung des Verhältnisses zwischen europäischer Integration und nationaler Identität im Auftrag der Nederlandsche Bank wurden die heutigen niederländischen Einstellungen über Europa gut zusammengefasst: „Die übergroße Mehrheit der Bevölkerung fühlt sich in erster Linie als Niederländer und mit dem eigenen Land verbunden. Die Niederlande sind das Bezugssystem, ,Europa ' ein Arbeitsgebiet. Niederländer wollen sich in der Europäischen Union, aber auch außerhalb als Niederländer darstellen. [...] Nur wenige streiten für ein Vereintes Europa mit einer eigenen Identität. [...] Niederländer betrachten Europa nicht so sehr mit Gefühlen, die ein neues Vaterland hervorruft, sondern sehen es eher als ein funktionelles und pragmatisches Modell der Zusammenarbeit, das dann relevant wird, wenn die eigenen, nationalen Belange dies erfordern. Die Föderalisten hatten die Hoffnung, Europa durch eine, ever closer union“ über die Nationalstaaten zu stellen. Sie scheiterten in den Niederlanden und in Frankreich an Bürgern, die bereits eine nationale Identität haben. Niederländer wollen nicht in den Vereinigten Staaten von Europa aufgehen." 12

\section{Eine Kluft zwischen Bürgern und europäischer Politik}

Angesichts der Tatsache, dass die Tweede Kamer mit großer Mehrheit für die europäische Verfassung war, ergibt sich in dieser Hinsicht eine deutliche Kluft zwischen Bürgern und Politik, eine gestörte Beziehung, die einem optimalen Funktionieren der niederländischen Demokratie innerhalb der Europäischen Union im Wege steht. Nach Meinung der Arbeitsgruppe

9 Jacques J.A. Thomassen: Nederlanders en Europa. Een bekoelde liefde?, in: Kees Aarts/Henk van der Kolk (Hrsg.): Nederlanders en Europa. Het referendum over de Europese grondwet, Amsterdam 2005, S. 66-67.

10 Paul Kapteyn/Bert Schijf: Europa, Ja, Nee, Geen Mening, Amsterdam 1996, S. 7.

11 Boris Dittrich/Sophie in 't Veld/Lousewies van der Laan: De Verenigde Staten van Europa. Pamflet voor een transparante, slagvaardige en toekomstgerichte Europese Unie, Den Haag 2005.

12 Corry van Renselaar/Ger Bom: Quo Vadis Europa? Europa: integratie versus nationale identiteit, Amsterdam 2006, S. 51-52. 
Europa des Nationalkonvents kann die gestörte Beziehung zwischen den niederländischen Bürgern und der europäischen Politik im Kern auf drei Übel zurückgeführt werden:

\section{Kaschierung tatsächlicher Interessenskonflikte}

Die Kluft, die am 1. Juni 2005 überdeutlich wurde, spiegelt vermutlich tatsächliche Interessensgegensätze in der niederländischen Gesellschaft wider. Einerseits gibt es politische, gesellschaftliche und finanz-wirtschaftliche Eliten, mit übrigens nicht wenig Rückhalt in der Bevölkerung, die großes Interesse an einer weitergehenden europäischen Integration haben und sich auch stark dafür einsetzen. Andererseits scheinen breite Schichten der niederländischen Bevölkerung diesen sehr zögerlich gegenüber zu stehen.

Es geht hier nicht um einen schlichten Gegensatz zwischen ,Elite' und ,Masse‘. Auch bei den Gegnern der europäischen Verfassung waren viele Akademiker, Menschen mit höherer Bildung, Journalisten und Beamte zu finden. ${ }^{13}$ Der Gegensatz betrifft nicht so sehr bestimmte Politikfelder (womit ein politisches System sehr gut leben kann), sondern einen grundlegenden Meinungsunterschied über den gewünschten Kurs der niederländischen Europapolitik und das Ausmaß, wie weit die nationale Souveränität an die Europäische Union abgegeben oder mit ihr geteilt werden soll.

Das Für und Wider weiterer europäischer Einigung betrifft vor allem die überaus wichtige politische Vorstellung der Entscheidungsbefugnis und nicht so sehr die verschiedenen spezifischen wirtschaftlichen Vor- und Nachteile. In dem Maße, in dem die Europäische Union sich auf breitere Politikfelder begeben hat, hat sich auch der Konflikt über die Mitspracherechte zugespitzt. In den ersten Jahren nach dem Krieg lag der Schwerpunkt unwidersprochen noch auf dem Bewahren von Frieden, dem Wiederaufbau und der wirtschaftlichen Zusammenarbeit als den zentralen Motiven für die europäische Integration. Zur Zeit mischt sich ,Brüssel ${ }^{\star}$ auch in Asylprobleme, Außenpolitik und BSE ein und scheint mehr und mehr eine Vielzahl von Aspekten des täglichen Lebens zu beeinflussen. Bei näherem Hinsehen scheint dieser Einfluss doch nicht so groß zu sein (die meisten Vorschriften sind überwiegend national geblieben), aber die Bürger haben einen anderen Eindruck. Die Ablehnung der europäischen Verfassung bezog sich nicht so sehr auf spezifische Teile des Vertrages (die so bahnbrechend nun auch nicht waren), sondern bildete eine Ablehnung zu weit gehenden europäischen Einflusses, wie er im europäischen Verfassungsprojekt symbolisiert wurde.

Für ein gutes Funktionieren des niederländisch-europäischen Systems ist es essenziell, dass dieser ziemlich prinzipielle Interessenskonflikt zwischen , mehr " und ,weniger 'Europa als solcher anerkannt und offen angesprochen wird. Die europäischen und nationalen Pressestellen haben zu sehr die Neigung, den Gegensatz zu maskieren und völlig vernünftige Beschwerden gegen fortgesetzten Brüsselschen Einfluss abzuqualifizieren. Beide ,Parteien“ (um das Schema weiter einfach zu halten) haben sehr legitime Interessen, die nur durch eine offene politische Debatte mit sich ins Reine kommen können. Letzteres ist nicht nur eine Frage besserer Kommunikation, vor allem, wenn es darum geht, diese Interessensgegensätze zu kaschieren. Darüber hinaus bedeutet mehr Information über die Europäische Union nicht automatisch, dass die Bürger die Europäische Union auch mehr würdigen. Je mehr Informa-

13 Aus einer Untersuchung der Zeitschrift Binnenlands Bestuur (vom 20. Mai 2005) am Vorabend des Referendums wurde deutlich, dass 44 Prozent der befragten niederländischen Beamten und leitenden Angestellten die Absicht hatten, gegen den Verfassungsvertrag zu votieren. Vgl. R.J.G.M. Widdershoven: De betekenis van de Europese grondwet voor het Nederlandse bestuur, in: H.-M.Th.D. ten Napel/W.J.M. Voermans (Hrsg.): De betekenis van de Europese Grondwet voor de Nederlandse staatsinstellingen, Deventer 2005, S. 39. 
tion die niederländischen Wähler während der Referendumskampagne über den Verfassungsvertrag bekamen, desto mehr stieg in den Umfragen der Anteil potenzieller Neinsager. Die Millionen „Nein“-Sager sind daher weniger ,unwissend“, als verschiedene Haager und Brüsseler Instanzen manchmal denken.

\section{Undeutliche Abgrenzung zwischen den Niederlanden und der Europäischen Union}

Ein zweites wichtiges Defizit ist nach Meinung des Nationalkonvents die überaus undurchsichtige und komplizierte europäisch-nationale Entscheidungsfindung, die für politische Partizipation ein Hindernis darstellt. Wenn Bürger nicht mehr wissen, wann und durch wen in welchen Politikbereichen Beschlüsse gefasst werden, wird ihr Interesse am politischen Prozess geringer werden, ebenso ihre Neigung, diesen Prozess zu beeinflussen. Der byzantinische Komplex aus europäischen und nationalen Einrichtungen, Räten, Arbeitsgruppen, Kommissionen, ,Komitologiekomitees" und dergleichen mehr fungiert in dieser Hinsicht als eine Art ,Morastbarriere` für ein ordentliches öffentliches Engagement in der europäischen Politik.

Vor allem die vertikale Abgrenzung der Zuständigkeiten (zwischen den Niederlanden und der Europäischen Union) erfahren viele Bürger als eine Quelle politischer Unsicherheit und Irritation. Dadurch bleibt die Legitimität der europäischen Einrichtungen (besonders die repräsentativen) deutlich hinter der des niederländischen Staates zurück.

\section{Fehlende Vision der politischen Finalité des europäischen Integrationsprozesses}

Ein drittes Defizit ist, dass der gewünschte Endzustand des europäischen Integrationsprozesses in den Niederlanden (und übrigens auch nicht in den meisten anderen EU-Mitgliedstaaten) nie (klar) definiert wurde. Die Niederlande sind seit mehr als einem halben Jahrhundert konstruktives Mitglied der Europäischen Gemeinschaften und der späteren Europäischen Union, aber die aufeinander folgenden Regierungen waren in all den Jahren überaus kopfscheu, eine Art politischer End- oder Zwischenstation für den europäischen Integrationsprozess zu formulieren, in welcher (kon)föderalen oder unitären Form auch immer. Anfänglich wurde wohl explizit über das Ideal eines „,öderalen“ Europas gesprochen, doch dieses Ideal ist nie ausgefüllt worden und starb nach dem Beginn der 1990er Jahre auch rhetorisch einen sanften Tod.

Die supranationalen Elemente in der Europäischen Gemeinschaft für Kohle und Stahl und in der Europäischen Wirtschaftsgemeinschaft wurden zunächst mit dem Argument gerechtfertigt, eine solche Gemeinschaft schließe Krieg zwischen den Beteiligten aus. Später ergab sich das System der Europäischen Wirtschaftsgemeinschaft aus den funktionellen Erfordernissen des Aufbaus und der Steuerung des Gemeinsamen Marktes. Auch die Hinzufügung des zweiten und dritten Pfeilers ging auf funktionelle Systembedürfnisse zurück. Eine wichtige Rolle spielte allerdings auch der Gedanke der Rechtsgemeinschaft.

Die niederländische Regierung hat sich jetzt dieses Mangels an politischer Finalität angenommen. Die Bürger haben das Gefühl, in einem dahin rasenden Zug ohne Bremsen zu sitzen. Sie haben mit großer Geschwindigkeit große europäische Projekte (Vollendung des Binnenmarktes, Einführung des Euro, Beitritt von zehn neuen Mitgliedstaaten, Entwurf einer europäischen Verfassung) zur Abstimmung vorgelegt bekommen und dieser Prozess scheint kein Ende zu finden. Immer noch treten neue Mitglieder bei. Die Verhandlungen mit der Türkei haben bereits begonnen. Es ist einfach, sich in der Analyse des Referendums wieder zu finden, die Atzo Nicolaï, Staatssekretär im Außenministerium, während einer Rede in Den Haag am 11. Juli 2005 machte: „Weil das Endziel der europäischen Integration bewusst 
aus der Diskussion heraus gehalten wurde, ist ihr [der Niederländer] Argwohn nur größer geworden. Vielleicht ist jetzt die Zeit gekommen, dieses Tabu zu brechen und in die Diskussion über dieses Endziel einzusteigen." 14 Der Nationalkonvent plädierte in diesem Zusammenhang dafür, die Europäische Union vorläufig als einen fest gefügten „Staatenverbund“ mit deutlichen Einschränkungen der Zuständigkeiten der Union anstelle einer mächtigen „Föderation“ zu definieren.

\section{Veränderungen in der europäischen Politik}

Die kritische Haltung der Niederländer zu Europa rührt nicht nur aus dem Widerstand gegen Brüssels wachsendes Bedürfnis, sich in alles einzumischen, her, sondern auch aus allerlei ziemlich einschneidenden gesellschaftlichen Veränderungen, die die Mitgliedstaaten zu einem nationaleren Kurs zwingen.

Seit Beginn der 1990er Jahre scheint der Nationalstaat durch die Demontage der Staatsmonopole, die Privatisierung der öffentlichen Dienstleistungen und die Globalisierung der Weltwirtschaft zurückzuweichen. Der Markt übernahm die Funktion der staatlichen Fürsorge, ein Prozess, an dem auch der europäische Binnenmarkt einen wichtigen Anteil hatte. Doch die negativen Auswirkungen von Globalisierung und Marktwirkung, wie zum Beispiel der Untergang der öffentlichen Dienstleistung in bestimmten Bereichen und der Umzug von Betrieben in Niedriglohnländer, haben den Ruf nach verstärktem Staatsauftreten wieder aufleben lassen.

Auch ein relativ neues europäisches Phänomen, nämlich der enorme Zustrom von (häufig bedürftigen) Einwanderern und Asylbewerbern, setzte den Staatsapparat in verschiedenen europäischen Ländern unter Druck, besonders in den Niederlanden, die eine verhältnismäßig hohe Einwanderungsquote haben. Das Entstehen ethnischer Ghettos in großen und mittelgroßen Städten, strukturelle Rückstände und Segregation im Bildungsbereich, hohe Arbeitslosigkeit und stark steigende Kriminalität weisen einer Organisation, die durch alle Liberalisierungs- und Privatisierungstendenzen zu verschwinden schien, eine Schlüsselrolle zu: dem nationalen Staat. Eine wirkungsvolle Politik für diese relativ neuen Problembereiche ist primär von speziellen Einrichtungen in den Mitgliedstaaten abhängig, von nationaler politischer Kultur, der Beziehung zwischen Bürgern und Staat, von Meinungen über die Rolle der Polizei und Strafrechtspflege, Bildungstraditionen, Finanzspielräumen und ähnlichem mehr. Diese Politik kann daher unmöglich mehr oder weniger harmonisiert von Brüssel aus dirigiert werden, auch wenn ein europäischer Rahmen in Bereichen wie Asyl, Einwanderung, Bekämpfung der organisierten Kriminalität etc. unerlässlich bleibt.

In dem Augenblick, in dem die Europäische Union ihr Verfassungsprojekt in Angriff nahm, forderten die Bürger nicht zu Unrecht von ihrem Staat eine nationale Antwort auf die neuen gesellschaftlichen Probleme, denen sie sich gegenüber sahen. Sie interessierten sich nicht für die europäische Rhetorik, in die sich ihre politische Führung öfter flüchtete. Und soweit sie kein Gehör fanden bei der etablierten Politik, bekamen populistische euroskeptische Politiker von selbst mehr Aufwind. Diese sind selbst weniger Anstifter der neuerlichen nationalen Orientierung in den verschiedenen Mitgliedstaaten als vielmehr das Produkt davon.

14 Atzo Nicolaï: Bescheiden, eerlijk en ambitieus. Naar een nieuw Europa voor de burgers, Den Haag 2005, abrufbar unter: www.minbuza.nl. (letzter Zugriff: 12.09.2007). Siehe auch H.-M.Th.D. ten Napel: Liever Monnet dan Metternich? De Haagse visie op het beginsel van het institutioneel evenwicht, in: H.-M. Th.D. ten Napel/ W.J.M. Voermans (Hrsg.): De betekenis van de Europese Grondwet voor de Nederlandse staatsinstellingen, Deventer 2005 (Publikaties van de staatsrechtkring 24 ), S. 19. 
Die Renationalisierungstendenz stellt die europäische Integration auf den Kopf. Die frühere Vorstellung, dass die europäische Integration notwendig ist, weil die nationale Kapazität nicht mehr ausreicht, greift nicht mehr. Im Gegenteil: die nationale Kapazität nimmt notwendigerweise auf Gebieten zu, auf denen die Europäische Union beinahe per definitionem wenig unmittelbar ausrichten kann. Auf diese Weise bleiben wesentliche Aspekte des politischen Lebens (öffentliche Ordnung, Haushalt, Legitimität, die Beziehung zwischen Bürgern und Staat) fest in ,nationalen ' Händen, auf Kosten einer europäischen politischen Identität.

Fortuyn, Haider oder Le Pen haben inzwischen das Feld geräumt. Die etablierten Parteien und Politiker haben allerdings deren ,nationale Agenda' zu einem guten Teil übernehmen müssen. Das hat nichts mit einem wiederauflebenden ideologischen Nationalismus nach Vorkriegsart zu tun, sondern mit einer notwendigen Neueinschätzung der Aufgaben des Staates in einem globalisierten System. Von einem schrittweisen transfer of loyalty, wie das frühere Ideal der europäischen Integration es wollte, kann unter solchen Umständen keine Rede mehr sein. Und diese Tatsache ist mitbestimmend für die restierende nationale Selbstständigkeit.

In den ersten Jahrzehnten nach dem Zweiten Weltkrieg hat der klassische Versorgungsstaat der europäischen Staatsbildung und einer europäischen Bürgerschaft im Weg gestanden. Heute passiert dies durch den postmodernen Staat, zwar in einem viel offeneren internationalen Umfeld, aber unvermeidlich oft mit den gleichen politischen, finanziellen und ideologischen Instrumenten. Die Arbeitsgruppe Europa des Nationalkonvents kommt zu dem Schluss, dass der europäische Integrationsprozess in Ländern wie den Niederlanden zu „wachsender nationaler Bewusstwerdung“ geführt hat. „Die Annahme, dass der politische Schwerpunkt nach Brüssel wandern würde, hat sich nicht bewahrheitet. Der Nationalismus ist im Allgemeinen überwunden, aber die politische Bedeutung des Nationalstaates bleibt unverändert groß, wenn auch maßvoller." 15

\section{Der Denkfehler der Verfassung}

Die Staatssymbolik des europäischen Verfassungsprojektes und zum Teil auch dessen Inhalt hat diese nationalen Kräfte in der heutigen europäischen Politik von Beginn an nur unzureichend berücksichtigt. Auf dem Papier sahen die Absichten gut aus: der Union größere Schlagkraft nach innen und außen verschaffen, die Möglichkeiten der Entscheidungsfindung durch qualifizierte Mehrheit erweitern, Verträge vereinfachen. Wie früher also sollte gelten: Erst Vertiefung, dann Erweiterung. Aber dieses Mal platzte der Ballon.

Die große Erweiterung (Big Bang) des Jahres 2004 konnte aus sich selbst heraus keine zwingende Rechtfertigung des Verfassungsabenteuers bilden, denn in Nizza wurden im Prinzip zunächst ausreichende institutionelle Vorkehrungen getroffen für eine EU-27, auch wenn viele davon enttäuscht waren. Die institutionellen Verbesserungen, die die europäische Verfassung brachte, waren wichtig, hätten aber auch noch warten können. Das Timing des Verfassungsprojektes war wenig glücklich, so kurz nach der Einführung des Euro und dem Beitritt weiterer zehn Mitglieder. Es gab keinen akuten funktionellen Grund dahinter, wie zum Beispiel bei der Einheitlichen Europäischen Akte (angesichts des Drucks für einen Binnenmarkt aus der europäischen Wirtschaft heraus), dem Vertrag von Maastricht (der die deutsche Vereinigung berücksichtigte) oder den Verträgen von Amsterdam und Nizza, die die große Ausbreitung der Union nach Osten und Süden mit vorbereiten sollten.

15 De Europese Unie als Statenverbond, 2006, S. 37. 
Und es fehlte eine Dimension, die auch unentbehrlich ist für ein modernes Verfassungsprojekt: ein breiter gesellschaftlicher Konsens in den Mitgliedstaaten, eine kraftvolle demokratische Bewegung aus Parteien und Lobbys, Kampagnen in Medien und Massendemonstrationen in den europäischen Hauptstädten. Von Beginn an war die europäische Verfassung ein klassisches Elitenprojekt, das von einigen enthusiastischen föderalistischen Politikern (wie dem deutschen Außenminister Joschka Fischer und dem belgischen Ministerpräsident Guy Verhofstadt) von oben durchgesetzt wurde und das eigentlich nur vom Europäischen Parlament aktiv unterstützt wurde. Der groß angelegte Europäische Konvent in Brüssel fand in den Mitgliedstaaten wenig öffentlichen Widerhall, obwohl er eine Vielzahl gesellschaftlicher Organisationen mit einbezog. Für große Teile der Bevölkerung handelte es sich um ein vollkommen unbekanntes Projekt, trotz der ziemlich offenen Konventmethode und der Tatsache, dass die Versammlung zum größten Teil aus Volksvertretern bestand (darunter der heutige Außenstaatssekretär Frans Timmermans).

Auf alle Fälle haben all diese Volksvertreter damals nicht gefühlt, was heute anscheinend doch in die europäischen politischen Kreise vorgedrungen ist, dass nämlich die Union noch nicht reif ist für ein Projekt mit deutlichen Verfassungsansprüchen. Viele Regierungen, die vor einigen Jahren die europäische Verfassung noch mit offenen Armen empfingen, müssen sich jetzt mit einem stark abgeschwächten Text begnügen, wie das Mandat für die Regierungskonferenz zeigt. Das französische und das niederländische „Nein“ haben die europäische Verfassungskrise nicht so sehr verursacht als vielmehr ans Licht gebracht. Beide Länder dürfen daher auch nicht als isolierte Fälle betrachtet werden. Die kritische Stimmung in der Öffentlichkeit gegen europäisches Machtstreben erstreckt sich auch auf andere Mitgliedstaaten.

\section{Das Europa der ,großen Würfe“" ist vorbei}

Es ist wichtig zu erkennen, dass durch das Scheitern der Verfassung die Perspektive weiterer politischer Integration vorläufig hinter dem Horizont verschwindet. Niemand will sich noch einmal ein Jahrzehnt über große Veränderungen im Aufbau der Union beugen und sich dann mit minimalen Resultaten begnügen müssen. Wenn 2008/9 ein neuer Vertrag unterzeichnet und ratifiziert wird, so wird das für geraume Zeit das letzte Mal in den allgemeinen Revisionszyklen von ungefähr fünf Jahren sein, die mit der Einheitlichen Europäischen Akte 1985/6 ihren Anfang nahmen - sicher solange die Einstimmigkeitsregel für Vertragsrevisionen gültig bleibt, wie im Mandat für die Regierungskonferenz festgelegt.

Das dynamische Konzept eines ,,immer engeren Verbundes“ von Staaten, die langsam aber sicher auf immer mehr Politikfeldern Zuständigkeiten an die europäischen Einrichtungen abgeben, hat für ein statischeres Integrationsmuster einer engen Staatengemeinschaft mit zum Teil übernationalen Einrichtungen, aber doch mit einem überwiegend intergouvernementalen Ansatz Platz gemacht: Eine Europäische Union, in der der Nachdruck auf den Mitgliedstaaten liegt und in der Zusammenarbeit und Integration auf Teilgebieten in wechselnder Zusammensetzung stattfinden.

Das Scheitern des europäischen Verfassungsprojektes markiert daher eine Wende im europäischen Integrationsprozess, unabhängig davon, was der Inhalt des neuen Reformvertrages sein wird. Über 50 Jahre hinweg bildete sich in den Niederlanden und darüber hinaus das Ideal einer föderalen politischen Union als Richtschnur für die europäische Integration (auch wenn diese nicht klar definiert wurde). Dieses Ideal, das aus den vielen grauenhaften Kriegserfahrungen auf dem europäischen Kontinent entstand, legitimierte und inspirierte sowohl den Aufbau der europäischen Einrichtungen und die Entwicklung neuer Politiken als 
auch die anhaltende Erweiterung der Union. Jetzt erfordert die europäische Realität ein anderes Bild der Zukunft und auch eine andere Legitimierung der europäischen Politik in den Niederlanden. ${ }^{16}$

\section{Die Regierung Balkenende IV und der neue Reformvertrag}

Alle diese Veränderungen im europäischen und niederländischen Integrationskontext haben inzwischen ihren Weg in Politik und Parlament gefunden. Dort ist in letzter Zeit größerer Nachdruck auf das Garantieren niederländischer Interessen und Identitätsmerkmale in der Europäischen Union zu finden. Dabei geht es nicht nur um unverfälschte nationale Töne aus den populistischen Kreisen der - inzwischen untergegangenen - Liste Pim Fortuyn (LPF), der Partei für die Freiheit (PVV) von Geert Wilders oder die in der Wählergunst stark wachsende Sozialistische Partei (SP).

Auch die großen, von alters her europäisch gesinnten Volksparteien der niederländischen Politik haben inzwischen einiges nationales Wasser in den europäischen Wein gegossen. Die rechtsliberale VVD von Frits Bolkestein machte dabei den Anfang, indem er in den 1990er Jahren unablässig auf eine Reduzierung der niederländischen Zahlungen an Brüssel pochte und sich sehr skeptisch über die politische Einigung Europas ausließ. Der spätere Kommissar Bolkestein gilt als der erste wichtige Euroskeptiker in der niederländischen Politik, ${ }^{17}$ der Schule gemacht hat bei seinen Nachfolgern wie Jozias van Aartsen und Gerrit Zalm. Nach Meinung des heutigen VVD-Vorsitzenden Mark Rutte muss man ,überaus zurückhaltend sein beim Aufgeben seiner [nationalen - Anmerkung des Verfassers] Vetorechte. Das tut man nur, wenn es nachweislich gut ist für das Wohlergehen der Niederlande, denn wir betrachten Europa in erster Linie als ein wirtschaftliches Projekt." 18

In der sozialdemokratischen PvdA hat sich das europäische Denken ebenfalls ziemlich in nationale Richtung verschoben. In einem Diskussionspapier, das diese Partei nach dem Referendum vorlegte, wird für eine Definition der europäischen Marktmechanismen, für das „Garantieren der öffentlichen Interessen durch die Mitgliedsstaaten“ und für eine Renationalisierung der EU-Politik plädiert. Die Arbeitsgruppe der PvdA will in diesem Zusammenhang ,nach Maßnahmen Ausschau halten, die jetzt europäisch geregelt sind, und von denen wir denken, dass diese am Besten auf nationale Ebene gehören." 19

Selbst die traditionell pro-europäische CDA (Christdemokraten) scheut das nationale Element nicht. Außenminister Bernard Bot (CDA) plädierte am Ende seiner Amtszeit im dritten Kabinett Balkenende für ,,mehr Nachdruck auf spezifische nationale Interessen im engeren Sinn“. In der heutigen Weltordnung muss seiner Meinung nach ein Land wie die Niederlande hart dafür arbeiten, dass „die ,Marke Niederlande“ im Ausland erhalten bleibt. “20 Bei den kleinen christlichen Parteien SGP und ChristenUnie bilden nationale Souveränität und Identität traditionell die Ausgangspunkte für eine europäische Zusammenar-

16 Der bereits oben erwähnte Bericht Europa in Nederland des Wissenschaftlichen Beirats der Regierung versucht, die Grundlage für diese neue Legitimationsart zu legen.

17 Sam Rozemond: Bolkestein en de Euroscepsis, Den Haag 1996. Für dieses Thema siehe auch Hans Vollaard/ Bartho Boer: Euroscepsis in Nederland, Utrecht 2005.

18 Interview Syp Wynia mit Mark Rutte: We hebben met een zeer links kabinet van doen, in: Elsevier, 7. Juli 2007.

19 Europa: vertrouwen herwinnen, Diskussionspapier der Arbeitsgruppe Europa der Partij van de Arbeid, Oktober 2005, S. 12.

20 Bernard Bot: Met overtuiging en berekening. Van zuiver naar realistisch multilateralisme, in: Internationale Spectator 11/2006, S. 547-551, hier: S. 547, 550. 
beit. ${ }^{21}$ Auch die links-liberale D66 übrigens will ihre eigenen sogenannten „Kronjuwelen“, die Errungenschaften auf dem Gebiet der Sterbehilfe, Schwangerschaftsabbruch und gleichgeschlechtliche Ehe, ausdrücklich aus dem Zugriff Brüssels heraus halten.

\section{Niederländische Nüchternheit}

Bei den Wahlen zur Tweede Kamer am 22. November 2006 behielten die etablierten Parteien per saldo eine komfortable Mehrheit (das ist wichtig für die parlamentarische Zustimmung zu einem folgenden europäischen Vertrag), aber die mehr oder weniger euroskeptischen Parteien auf der linken wie rechten Seite des parlamentarischen Spektrums (Partij voor de Vrijheid - PVV, Socialistische Partij - SP, ChristenUnie) gewannen viele Sitze hinzu. Die neue Regierungskoalition aus CDA, Partij van de Arbeid - PvdA und ChristenUnie, die das vierte Kabinett unter Ministerpräsident Balkenende bildet, ist sich über diese Akzentverschiebung im Klaren. In ihrem im Februar 2007 geschlossenen Koalitionsvertrag bildet eine ,aktive internationale und europäische Rolle“ zwar den erstgenannten Pfeiler (von sechs), aber der Ausdruck europäische Integration hat in diesem Vertrag systematisch Platz gemacht für die viel schwächere „europäische Zusammenarbeit“. Auch ist die allgemeine Tonlage bezüglich der Zukunft der Europäischen Union sehr zurückhaltend. ${ }^{22}$

Im März 2007 haben die neuen Regierungsmitglieder Maxime Verhagen (CDA, Außenminister) und Frans Timmermanns (PvdA, Außenstaatssekretär) dem Parlament einen Brief zur Vorbereitung der Vertragsrevisionen zukommen lassen, die die verworfene europäische Verfassung ersetzen sollen. Darin setzen sie starke nationale Akzente: ,So wie alle anderen Europäer identifizieren sich die Niederländer im Allgemeinen in erster Linie mit ihrem eigenen Land. Dort liegt der Fokus der politischen Interessen und der politischen Diskussion. Deshalb muss die politische Priorität der Mitgliedstaaten bei der Gestaltung der gemeinsamen europäischen Zukunft auch deutlich markiert werden. Ein neuer Vertrag muss das berücksichtigen. Die Benennung und die Form des Verfassungsvertrages sowie bestimmte symbolische und inhaltliche Elemente weckten bei vielen Bürgern den Eindruck eines Sprunges in die Richtung eines europäischen Staates, der sich über oder an die Stelle des nationalen Staates stellen würde. Ein neuer Vertrag darf nicht die Züge einer Verfassung haben. Es muss deutlich sein, dass die Europäische Union keine autonome Organisation (ein ,Superstaat") wird, sondern dass die Europäische Union nur Zuständigkeiten ausüben kann, die ihr die Mitgliedsstaaten nach gemeinsamem Beschluss übertragen haben. “23

Dies ist eine zutreffende Zusammenfassung der heutigen niederländischen Nüchternheit in Europa. Fort mit der europäischen Hymne, einer europäischen Flagge, einem europäischen Außenminister und dem prominenten Platz für die Charta der Grundrechte, die im abgelehnten Text wie eine Bill of Rights präsentiert worden war.

Die Regierung will weiter verhindern, dass die EU-Gesetzgebung ,direkt oder indirekt Hindernisse aufwirft für unsere nationalen sozialen Arrangements oder für die Qualität der öffentlichen Dienste“. Es muss eine schärfere Abgrenzung geben zwischen nationalen und europäischen Zuständigkeiten, vor allem auf Gebieten, die „,wie keine anderen in der Hauptsache eine nationale Aufgabe sind, wie zum Beispiel Rentensysteme, soziale Sicherheit,

21 Dabei muss man sich darüber im Klaren sein, dass die Anschauung der ChristenUnie erheblich europäischer (geworden) ist als die der Staatskundige Gereformeerde Partij (SGP). Vgl. Benjamin Anker/Sander Luitwieler: Richting Europa: christelijk staatkundige visie op de Europese Unie, Amersfoort 2002; L. de Waal u.a.: Boodschap aan Europa: SGP-visie op de Europese Unie, Den Haag 2003.

22 Koalitionsvertrag zwischen den Parlamentsfraktionen von CDA, PvdA und ChristenUnie, 7. Februar 2007.

23 Kamerbrief inzake EU-verdragswijziging, ministerie van Buitenlandse zaken, 19. März 2007. 
Steuern, Kultur, Bildung und Gesundheit“".24 Die nationalen Parlamente müssen nach Meinung der Regierung mehr Mitsprache erhalten bezüglich Subsidiarität und auf diesem Gebiet eventuell auch eine ,rote Karte“ gegen zu weit gehende Vorschläge der Kommission ziehen können. Staatssekretär Frans Timmermans ließ in einem Vortrag an der Berliner Humboldt-Universität am 21. Mai 2007 keinen Zweifel darüber, dass seiner Meinung nach „die nationalen Parlamente der Fokus der politischen Interessen und der politischen Debatte von Europäern sind - und nicht Brüssel oder Straßburg“".25 Ungewohnte Töne in einem Land, in dem über viele Jahre hinweg ein stärkeres Europäisches Parlament als eines der „Fenster“ im europäischen Integrationskanon betrachtet wurde. ${ }^{26}$

\section{Ein Referendum?}

Es bleibt ein heikler Punkt für die niederländische Europapolitik in der nächsten Zeit: erfordern die anstehenden Überarbeitungen der europäischen Verträge ein neues Referendum? Die Regierung ist in diesem Punkt uneins. Die CDA ist entschieden dagegen, aber die PvdA hat in ihrem Programm für die Parlamentswahlen im November 2006 eine solche Volksbefragung versprochen, auch wenn der Reformvertrag keinen deutlichen Verfassungscharakter mehr haben sollte. Der Fraktionsvorsitzende der PvdA in der Tweede Kamer hat in einer Reaktion auf das Mandat für die Regierungskonferenz im Juni 2007 erneut dafür plädiert, dies im Gegensatz zum Koalitionsvertrag, der ein Urteil darüber zunächst dem Raad van State (Staatsrat) überlassen will, dem höchsten juristischen Beratungsgremium der Regierung. Die PvdA fühlt sich allerdings zunehmend unter Druck gesetzt durch die stark aufkommende euroskeptische Sozialistische Partei.

Inzwischen hat die Regierung den Staatsrat im Juli 2007 um eine Art Vorgutachten zum Regierungskonferenz-Mandat gebeten, ein unüblicher Schritt (es gibt ja noch keinen Text eines Vertragsentwurfes), aber die Regierung will anscheinend einen frühzeitigen Aufschluss haben, wahrscheinlich auch wegen des Drucks der europäischen Partner. In der Europäischen Union fürchtet man nämlich die niederländischen Ratifizierungsquerelen.

Wohin der Würfel rollen wird, ist zum Zeitpunkt der Niederschrift dieses Textes noch unklar. Die Regierung Balkenende hat am 21. September 2007 bekannt gegeben, keinesfalls ein neues Referendum zu beabsichtigen. Mehrere Oppositionsparteien in der Tweede Kamer haben bereits einen Initiativantrag angekündigt, um eine Volksabstimmung trotzdem möglich zu machen. Sowohl Befürworter als auch Gegner haben gute Argumente. Die Gegner eines Referendums können zu Recht darauf verweisen, dass der Reformvertrag, auf den das Mandat für die Regierungskonferenz verweist, tatsächlich von allerlei Verfassungselementen bereinigt wird. Und das ist nicht nur eine kosmetische Operation, wie verschiedene Oppositionsmitglieder zu Unrecht vorbrachten. Das Verfassungselement stellte einen bewussten und wesentlichen Teil des abgelehnten Vertrages dar und mit der Auslassung dieses Teils verliert der neue Vertrag in der Tat eine sensible politische Dimension, trotz der vielen

24 Nach dem am 21./22. Juni 2007 ergangenen Mandat für die neue Regierungskonferenz zu urteilen haben die Haager Unterhändler diese Gesichtspunkte in EU-Kreisen erfolgreich durchsetzen können. Für den Text dieses Mandats siehe Schlussfolgerungen des Vorsitzes des Europäischen Rates von Brüssel, 21./22. Juni 2007, Dok. 11177/07 vom 23. Juni 2007.

25 Frans Timmermans: Das Europa der Anderen, Vortrag an der Humboldt-Universität, Berlin, 21. Mai 2007.

26 So plädierte der im Juli 2007 verstorbene Schelto Patijn in seiner 1973 erschienenen Dissertation über das Europäische Parlament für genau das Gegenteil dessen, was sein Parteikollege Frans Timmermans jetzt forderte. Nach Patijn muss das Europäische Parlament im Zentrum der Entwicklung der europäischen Demokratie stehen: „Eines ist sicher: es kann nicht mehr lange gewartet werden mit der Einführung der parlamentarischen Demokratie in die Europäischen Gemeinschaften." Schelto Patijn: Het Europees Parlement. De strijd om zijn bevoegdheden, Rotterdam 1973, S. 173. 
institutionellen Bestimmungen, die geblieben sind. Eine normale parlamentarische Zustimmungsprozedur kann hier im Prinzip ausreichen, wie es Premierminister Balkenende will.

Dennoch gibt es ein Problem. Denn was übrig bleibt, kann sicher nicht marginal genannt werden. Das gilt zum Beispiel für den festen Vorsitz im Europäischen Rat, die juristische Festschreibung der Charta der Grundrechte, die Erweiterung der Zuständigkeiten des Europäischen Parlamentes oder die Bestimmungen über Umfang und Zusammenstellung der Europäischen Kommission. Sicher ist auch, dass die Beschlussfassung durch qualifizierte Mehrheit auf vielen Gebieten erheblich ausgeweitet werden soll. Auch der Hohe Vertreter der Union für Außen- und Sicherheitspolitik erhält durch die Doppelfunktion als Vizepräsident der Europäischen Kommission und ständiger Vorsitzender des Rates für Auswärtige Angelegenheiten deutlich mehr Zuständigkeiten, auch wenn er oder sie sich nicht Außenminister nennen darf.

Die zweite Regierung Balkenende sprach bei der Vorstellung der europäischen Verfassung von einer „gründlichen Überarbeitung“, von „,bahnbrechenden Schritten“ und „hohem Anspruchsniveau“. Daher bezeichnete sie den Begriff Verfassung auch als ,gerechtfertigt“, obwohl sie selbst nicht für ein Referendum war. ${ }^{27}$ Als aber praktisch die gleichen Punkte in praktisch der gleichen Form (nämlich eines Vertrages) erneut ins Feld geführt wurden, waren sie plötzlich nicht mehr ,grundlegend“ und „bahnbrechend“? Derartige Qualifizierungen können nicht dadurch eliminiert werden, indem der Text etwas ausgedünnt und ihm ein anderes Etikett aufgeklebt wird.

Es gibt gute Gründe dafür, kein weiteres Referendum abzuhalten: es passt nicht in die niederländische parlamentarische Tradition, die Texte sind zu kompliziert für ein einfaches Ja oder Nein, ein weiteres „Nein“ wäre eine Katastrophe für die niederländische Zukunft in Europa. Der Ruf der Niederlande und ihre Verhandlungsposition in der Europäischen Union haben sich in den letzten zwei Jahren ohnehin verschlechtert. Im Nachhinein betrachtet hätte es niemals so weit kommen dürfen. Aber ein deutliches „Nein“ einer direkten Volksbefragung kann nicht dadurch ungeschehen gemacht werden, indem das Volk jetzt nicht mehr direkt befragt wird. Das würde die ,misstrauische Hellhörigkeit“ in der Bevölkerung sicher noch einmal wecken. ${ }^{28}$

Die beste Art und Weise, die Hypothek des „Nein“ zu tilgen, ist, dafür Sorge zu tragen, beim nächsten Mal ein deutlich vernehmbares „Ja“ für einen praktisch ausführbaren, politisch gut eingegrenzten Vertrag folgen zu lassen. Die Regierung muss die Möglichkeit eines fakultativen (nicht verpflichtenden), aktiven (von der Bevölkerung initiiert) und beratenden (formal nicht bindend, wohl aber politisch bindend) Referendums offen halten. Ein großer Vorteil ist, dass ein solches Referendum die europäischen Angelegenheiten in den Niederlanden hoch auf die Agenda setzt und so zu einer breiten gesellschaftlichen Diskussion führt. Das Ja-Lager kann sich gut revanchieren dadurch, dass man jetzt rechtzeitig beginnt und die Intelligenz des niederländischen Volkes nicht länger unterschätzt mit bedrohlichen Reden über den Untergang Europas. Ein klares „Ja“ stellt sowohl das niederländische Ansehen in

27 Die zweite Regierung Balkenende urteilte auf der Grundlage verschiedener Punkte, die auch im Mandat für die Regierungskonferenz vorkommen: „Kurzum, der Verfassungsvertrag zeigt eine derartige Übereinstimmung mit nationalen Verfassungen, dass, auf alle Fälle in politischem Sinn, von einer Verfassung gesprochen werden kann." Tweede Kamer der Staten Generaal, Vergaderjaar 2005-2005, Goedkeuring van het op 29 oktober 2004 te Rome tot stand gekomen Verdrag tot Vaststelling van een Grondwet voor Europa, 30025 (R 1783), Memorie van Toelichting, S. 5.

28 Der Ausdruck stammt von Monika Sie Dhian Ho in De Volkskrant, 1. Juni 2006. Siehe auch Marcel Becker/ Kees Klop/Bas van Stokkom/Jean-Pierre Wils (Hrsg.): Na het referendum over Europa: een weg uit de impasse, Budel 2006. Vgl. Mark Kranenburg: "Nederland, Europa, en het nee tegen de Europese Grondwet", in: C.C. van Baalen u.a. (Hrsg.): Jaarboek Parlementaire Geschiedenis 2006, Amsterdam 2006, S. 85. 
Europa als auch das Ansehen Europas in den Niederlanden wieder her. Im Bericht der Nederlandsche Bank heißt es zu Recht: „Die Brüsselsche Macht ist groß, nach Meinung vieler Niederländer zu groß. Die Übertragung weiterer Zuständigkeiten stößt auf breiten Widerstand, vor allem, wenn dies geschieht, ohne dass die Bevölkerung ihre Stimme hören lassen kann." $" 29$

Alles in Allem sind die jüngsten Veränderungen in der niederländischen Europapolitik also ziemlich einschneidend, sowohl in der Politik als auch in der Bevölkerung. Anstelle eines (vagen) föderalen Ideals als Richtschnur sucht die Regierung jetzt eher sachliche politische und wirtschaftliche Argumente für die weitere Integration. Der Fortschreibung der Rolle des nationalen Staates wird viel Gewicht beigemessen. Und anstelle der stillschweigenden Zustimmung zur Haager Europapolitik gibt es jetzt viel schneller kritisches Interesse in der breiten Öffentlichkeit. Die traditionelle Europaelite hat ihren alleinigen Anspruch verloren. Darüber darf nicht vergessen werden, dass die Niederlande noch immer in allen Bereichen der europäischen Integration und auf allen Gebieten der drei Unionspfeiler beteiligt sind. Die grundsätzliche Unterstützung für das europäische Projekt bleibt bestehen, solange dieses nicht zu sehr in die niederländische politische Ordnung eingreift.

Übersetzung aus dem Niederländischen von Gudrun Staedel-Schneider.

29 Van Renselaar/Bom: Quo vadis Europa, 2006, S. 53. 